\title{
One-pot, three component reactions between isocyanides and dialkyl acetylenedicarboxylates in the presence of phenyl isocyanate: synthesis of dialkyl 2-(alkyl/arylimino)-2,5-dihydro-5- oxo-1-phenyl-1H-pyrrole-3,4-dicarboxylate
}

\author{
Khatereh Khandan-Barani, Malek T. Maghsoodlou,* Nourallah Hazeri, \\ Sayyed M. Habibi-Khorasani, and Seyed Sajad Sajadikhah \\ Department of Chemistry, University of Sistan and Baluchestan, \\ PO Box 98135-674, Zahedan, Iran \\ E-mail: mt_maghsoodlou@yahoo.com
}

DOI: http://dx.doi.org/10.3998/ark.5550190.0012.b02

\begin{abstract}
The reactive 1:1 adduct, generated from the reaction between alkyl/aryl iscyanides and dialkyl acetylenedicarboxylates, was trapped by phenyl isocyanate to yield dialkyl 2-(alkyl/arylimino)2,5-dihydro-5-oxo-1-phenyl-1H-pyrrole-3,4-dicarboxylate in good yields.
\end{abstract}

Keywords: Phenyl isocyanate, 2,5-dihydro-5-oxo-1-phenyl-1H-pyrrole derivatives, three component reactions, isocyanides, acetylenic esters

\section{Introduction}

Pyrrole derivatives are an important class of heterocycles. Nitrogen heterocycles are of synthetic interest because they constitute an important class of natural and non-natural product, many of which exhibit useful biological activity. ${ }^{1}$

Isocyanide-based multicomponent reactions (IMCR) now occupy a position of importance in synthetic organic chemistry, mainly due to the contributions of Ugi and co-workers. ${ }^{2}$ Organic isocyanates are powerful tools in organic synthesis. ${ }^{3-5}$ Generally, isocyanates easily undergo polar cycloadditions with a large variety of unsaturated substrates. ${ }^{6-7}$

The reactivity of nucleophilic carbenes such as isocyanides towards dimethyl acetylenedicarboxylate (DMAD) is well documented..$^{8-10}$ The reaction of isocyanides with carbon- carbon triple bonds occurs in a stepwise manner through a zwitterionic intermediate, the ultimate fate of which appears to be dictated by the nature of original triple-bonded substrate. ${ }^{11-19}$ In continuation of our interest in the application of isocyanides in heterocyclic synthesis, ${ }^{20-27}$ we 
now report the reaction between alkyl/aryl isocyanides $\mathbf{1}$ and dialkyl acetylenedicarboxylate $\mathbf{2}$ in the presence of phenyl isocyanate. This one-pot, three component synthesis proceeded spontaneously at $38{ }^{\circ} \mathrm{C}$ in $\mathrm{CH}_{2} \mathrm{Cl}_{2}$ and leads to dialkyl 2-(alkyl/arylimino)-2,5-dihydro-5-oxo-1phenyl-1H-pyrrole derivatives 3a-h (Scheme 1).

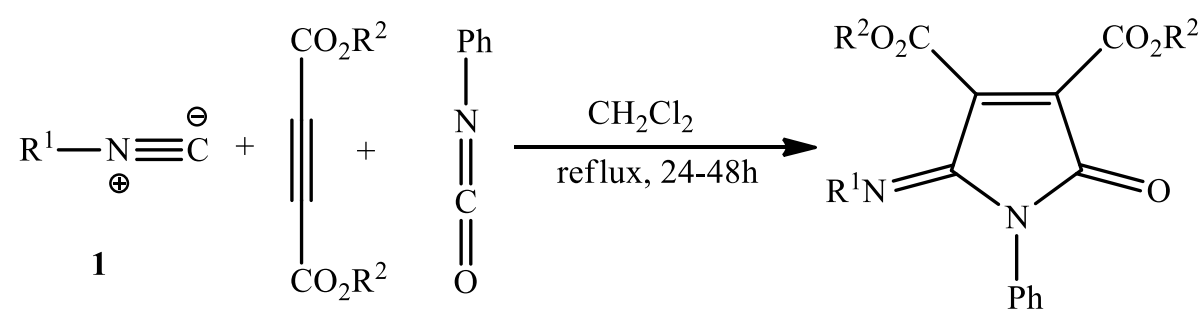

2

3a-h

\begin{tabular}{l|lr|l}
1 & $\mathrm{R}^{1}$ & 2 & $\mathrm{R}^{2}$ \\
\hline $\mathrm{a}$ & Cyclohexyl & $\mathrm{a}$ & $\mathrm{Me}$ \\
$\mathrm{b}$ & $t$-butyl & $\mathrm{b}$ & $\mathrm{Et}$ \\
$\mathrm{c}$ & 2,6 -Dimethylphenyl & $\mathrm{c}$ & $t$-Bu
\end{tabular}

\begin{tabular}{l|l|c|l|l}
3 & $\mathrm{R}^{1}$ & $\mathrm{R}^{2}$ & Time $(\mathrm{h})$ & Yield (\%) \\
\hline $\mathrm{a}$ & Cyclohexyl & $\mathrm{Me}$ & 24 & 93 \\
$\mathrm{~b}$ & Cyclohexyl & $\mathrm{Et}$ & 36 & 95 \\
$\mathrm{c}$ & Cyclohexyl & $t$-Bu & 48 & 88 \\
$\mathrm{~d}$ & $t$-butyl & $\mathrm{Me}$ & 30 & 95 \\
$\mathrm{e}$ & $t$-butyl & $\mathrm{Et}$ & 36 & 82 \\
$\mathrm{f}$ & $t$-butyl & $t$-Bu & 36 & 85 \\
$\mathrm{~g}$ & 2,6-Dimethylphenyl & $\mathrm{Me}$ & 24 & 84 \\
$\mathrm{~h}$ & 2,6-Dimethylphenyl & $t$-Bu & 48 & 81
\end{tabular}

Scheme 1. Synthesis of compounds 3a-h.

\section{Result and Discussion}

The structure of compounds 3a-h was deduced from their IR, ${ }^{1} \mathrm{H}$ NMR, ${ }^{13} \mathrm{C}$ NMR, mass spectral data, HRMS data and elemental analysis. The mass spectra of these compounds 3a-h displayed molecular ion peaks at appropriate $m / e$ values. The ${ }^{1} \mathrm{H}$ NMR spectrum of compound 3a exhibited a multiplet for the five $\mathrm{CH}_{2}$ of cyclohexyl ring $(\delta 1.49-1.81 \mathrm{ppm})$, a multiplet for the $\mathrm{N}-\mathrm{CH}$ cyclohexyl proton $(\delta 3.78 \mathrm{ppm})$, two singlet for the methoxy groups ( $\delta 3.96$ and $3.99 \mathrm{ppm})$. The aromatic hydrogens gave rise to characteristic multiplet signal in the aromatic region of the spectrum $(\delta 7.23-7.43 \mathrm{ppm})$.

The ${ }^{13} \mathrm{C}$ NMR spectrum of $3 \mathbf{a}$ showed sixteen distinct resonances in agreement with proposed structure. The characteristic signal due to the amide carbonyl group was discernible at $\delta 148.0 \mathrm{ppm}$. Carbons of imino and two ester carbonyls resonated at $\delta 147.6,160.3$ and 160.6 ppm, respectively. Partial assignment of these resonances is given in the experimental data.

Dimethyl 2-(cyclohexylimino)-2,5-dihydro-5-oxo-1-phenyl-1 $H$-pyrrole-3,4-dicarboxylate 3a was obtained as a orange powder. Its molecular formula, $\mathrm{C}_{20} \mathrm{H}_{22} \mathrm{~N}_{2} \mathrm{O}_{5}$, was determined on the basis of the positive HRESIMS at $m / z 393.1435[\mathrm{M}+\mathrm{Na}]^{+}$(calcd 393.1426) and supported by the ${ }^{1} \mathrm{H},{ }^{13} \mathrm{C}$ NMR and IR spectra. 
The IR spectra of 3a showed strong absorptions at 1718 and $1750 \mathrm{~cm}^{-1}$ due to the ester carbonyls and also at $1656 \mathrm{~cm}^{-1}$ because of the carbonyl of amide. The ${ }^{1} \mathrm{H}$ and ${ }^{13} \mathrm{C}$ NMR spectra of $\mathbf{3 b} \mathbf{b}-\mathbf{h}$ are similar to $\mathbf{3 a}$ and the results are described in experimental section.

Mechanistically, it is conceivable that the reaction involves the initial formation of a 1:1 zwitterionic intermediate $\mathbf{4}$ in accord with reaction between alkyl/aryl isocyanide $\mathbf{1}$ and dialkyl acetylenedicarboxylate 2 . Nucleophilic addition of $\mathbf{4}$ to the carbonyl group of phenyl isocyanate formed a dipolar species 5. Cyclization of the latter leads to the 2,5-dihydro-5-oxo-1-phenyl-1Hpyrrole derivatives 3 (Scheme 2).

In this work, we used two factors to generated pyrrole derivatives, first, temperature factor by refluxing in $\mathrm{CH}_{2} \mathrm{Cl}_{2}$ at $38{ }^{\circ} \mathrm{C}$ and the second, time factor between $24-48$ hours. Therefore we have developed a one-pot three-component reaction between alkyl/aryl isocyanides $\mathbf{1}$ and dialkyl acetylenedicarboxylate 2 in the presence of phenyl isocyanate for the preparation of dialkyl 2(alkyl/arylimino)-2,5-dihydro-5-oxo-1-phenyl-1H-pyrrole derivatives 3a-h.

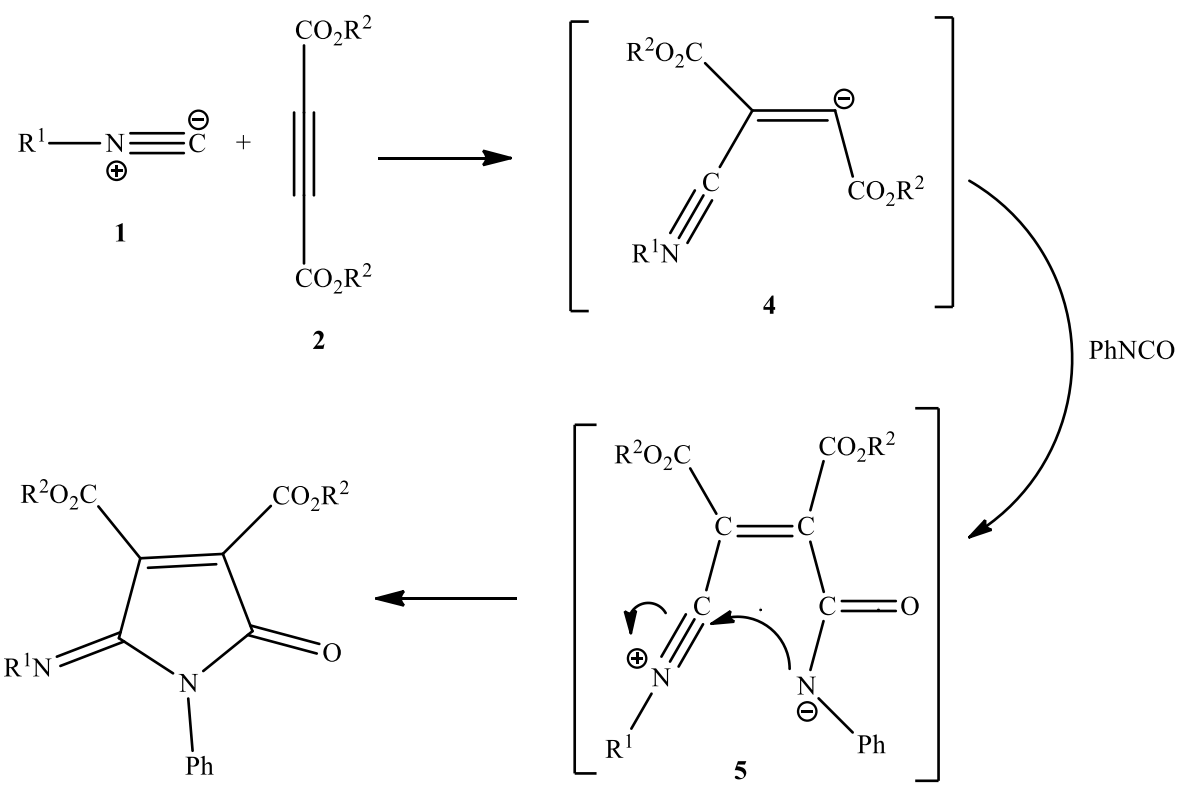

3

Scheme 2. Proposed mechanism for the formation of compound 3.

\section{Conclusions}

In summary, we have found that the reaction of alkyl/aryl isocyanides with dialkyl acetylenedicarboxylates in the presence of phenyl isocyanate leads to the one-pot and simple synthesis of highly functionalized 2,5-dihydro-5-oxo-1-phenyl-1H-pyrrole derivatives. The present method carries the advantages that, not only is the reaction performed under neutral conditions, but the substances can be mixed without any activation or modification. 


\section{Experimental Section}

General. Cyclohexyl isocyanide, $t$-butyl isocyanide, 2,6-dimethylphenyl isocyanide, dialkyl acetylenedicaboxylates and phenyl isocyanate were purchased from Fluka and Aldrich and used without further purification. Melting points and IR spectra were measured on an Electrothermal 9100 apparatus and a JASCO FT-IR spectrometer, respectively. The ${ }^{1} \mathrm{H}$ and ${ }^{13} \mathrm{C}$ NMR spectra were recorded on a BRUKER DRX-400 and 500 AVANCE instrument with $\mathrm{CDCl}_{3}$ as solvent at 400.1 MHz ( ${ }^{1} \mathrm{H}$ NMR), 100.6 and $125.7 \mathrm{MHz}\left({ }^{13} \mathrm{C}\right.$ NMR). Mass spectra were recorded on a Shimadzu GC/MS QP 1100 EX mass spectrometer operating at an ionization potential of $70 \mathrm{eV}$. Elemental analyses for $\mathrm{C}, \mathrm{H}$ and $\mathrm{N}$ using a Heraeus CHN-O-Rapid analyser were carried out at the Iranian Central Research of Petroleum Company. High resolution mass spectra (HRMS) were recorded on a Waters LCT Premier XW (ESI-TOF MS) spectrometer at Research School of Chemistry, Australian National University, Canberra ACT 0200, Australia.

\section{General synthetic procedure (exemplified by 3a)}

The solution of cyclohexyl isocyanide $(0.13 \mathrm{~g}, 1.2 \mathrm{mmol})$ in $3 \mathrm{~mL}$ of $\mathrm{CH}_{2} \mathrm{Cl}_{2}$ solvent was slowly added dropwise to a mixture of phenyl isocyanate $(0.12 \mathrm{~g}, 1 \mathrm{mmol})$ and DMAD $(0.17 \mathrm{~g}, 1.2$ $\mathrm{mmol}$ ) in $20 \mathrm{~mL}$ of $\mathrm{CH}_{2} \mathrm{Cl}_{2}$ solvent for $3 \mathrm{~min}$ at room temperature. After the addition, the solution was refluxed at $38{ }^{\circ} \mathrm{C}$ for $24 \mathrm{~h}$. Then, the solvent was removed under reduced pressure, and the solid product washed with mixture of cold diethyl ether and n-hexane with 1: 3 ratio $(2 \times 3 \mathrm{~mL})$. The liquid phase was filtered off and residual recrystallized in diethyl ether.

Dimethyl 2-(cyclohexylimino)-2,5-dihydro-5-oxo-1-phenyl-1H-pyrrole-3,4-dicarboxylate (3a). Orange powder, yield 93\%, $0.34 \mathrm{~g}, \mathrm{mp} 64-66{ }^{\circ} \mathrm{C}$; IR $(\mathrm{KBr})\left(v_{\max }, \mathrm{cm}^{-1}\right): 1750$ and 1718 ( $2 \mathrm{C}=\mathrm{O}$ of ester), $1656(\mathrm{C}=\mathrm{O}) .{ }^{1} \mathrm{H}$ NMR $\left(400.1 \mathrm{MHz}, \mathrm{CDCl}_{3}\right): \delta_{\mathrm{H}} 1.49-1.81\left(10 \mathrm{H}, \mathrm{m}, 5 \mathrm{CH}_{2}\right), 3.78$ $(1 \mathrm{H}, \mathrm{m}, \mathrm{NCH}), 3.96$ and $3.99\left(6 \mathrm{H}, 2 \mathrm{~s}, 2 \mathrm{OCH}_{3}\right), 7.23-7.43(5 \mathrm{H}, \mathrm{m}, \mathrm{Ar}-\mathrm{H}) .{ }^{13} \mathrm{C} \mathrm{NMR}(125.7 \mathrm{MHz}$, $\left.\mathrm{CDCl}_{3}\right): \delta_{\mathrm{C}} 24.4,25.5$ and $33.4\left(5 \mathrm{CH}_{2}\right.$ of cyclohexyl), 53.1 and $53.2(2 \mathrm{OMe}), 58.6(\mathrm{~N}-\mathrm{CH})$, $124.8,126.6,128.7,136.3,137.9,144.0\left(\mathrm{C}_{\text {arom }}\right.$ and $\left.\mathrm{C}=\mathrm{C}_{\text {pyrrole ring }}\right), 147.6(\mathrm{C}=\mathrm{N}), 148.0(\mathrm{C}=\mathrm{O})$, 160.3 and $160.6\left(2 \mathrm{C}=\mathrm{O}\right.$ of ester). $\mathrm{MS}$, we $(\%)=371\left(\mathrm{M}^{+}+1,16\right), 370\left(\mathrm{M}^{+}, 4\right), 338(100), 308$ (86), 252 (12), 197 (65), 119 (45), 83 (18), 77 (48), 55 (82); HRMS (ESI): $m z=[\mathrm{M}+\mathrm{Na}]^{+} \mathrm{Calcd}$ for $\mathrm{C}_{20} \mathrm{H}_{22} \mathrm{~N}_{2} \mathrm{O}_{5} \mathrm{Na}$, 393.1426; Found: 393.1435.

Diethyl 2-(cyclohexylimino)-2,5-dihydro-5-oxo-1-phenyl-1H-pyrrole-3,4-dicarboxylate (3b). Yellow powder, yield: $95 \%, 0.38 \mathrm{~g}, \mathrm{mp} 63-65^{\circ} \mathrm{C}$; IR $(\mathrm{KBr})\left(v_{\max }, \mathrm{cm}^{-1}\right): 1744$ and $1703(2 \mathrm{C}=\mathrm{O}$ of ester), $1660(\mathrm{C}=\mathrm{O}) .{ }^{1} \mathrm{H} \mathrm{NMR}\left(400.1 \mathrm{MHz}, \mathrm{CDCl}_{3}\right): \delta_{\mathrm{H}} 1.38$ and $1.41\left(6 \mathrm{H}, 2 \mathrm{t},{ }^{3} \mathrm{~J}_{\mathrm{HH}}=7.1 \mathrm{~Hz}\right.$, $\left.2 \mathrm{CH}_{3}\right), 1.11-1.93\left(10 \mathrm{H}, \mathrm{m}, 5 \mathrm{CH}_{2}\right), 3.77(1 \mathrm{H}, \mathrm{m}, \mathrm{NCH}), 4.42$ and $4.44\left(2 \mathrm{q},{ }^{3} \mathrm{~J}_{\mathrm{HH}}=7.1 \mathrm{~Hz}\right.$, $\left.2 \mathrm{OCH}_{2}\right), 7.23-7.54(5 \mathrm{H}, \mathrm{m}, \mathrm{Ar}-\mathrm{H}) .{ }^{13} \mathrm{C} \mathrm{NMR}\left(125.7 \mathrm{MHz}, \mathrm{CDCl}_{3}\right): \delta_{\mathrm{C}} 13.9$ and $14.0\left(2 \mathrm{CH}_{3}\right)$, 24.3, 25.6 and $33.4\left(5 \mathrm{CH}_{2}\right.$ of cyclohexyl), $58.5(\mathrm{~N}-\mathrm{CH}), 62.4$ and $62.4\left(2 \mathrm{OCH}_{2}\right), 124.9,126.6$, 128.7, 135.9, 137.7, $144.1\left(\mathrm{C}_{\text {arom }}\right.$ and $\left.\mathrm{C}=\mathrm{C}_{\text {pyrrole ring }}\right), 147.8(\mathrm{C}=\mathrm{N}), 148.2(\mathrm{C}=\mathrm{O}), 159.8$ and 160.1 $\left(2 \mathrm{C}=\mathrm{O}\right.$ of ester); MS, $m$ e $(\%)=398\left(\mathrm{M}^{+}, 2\right), 352$ (100), 325 (19), 306 (93), 301 (6), 279 (31), 252 (19), 197 (59), 119 (58), 83 (19), 77 (62), 55 (75); HRMS (ESI): $m z=[\mathrm{M}+\mathrm{Na}]^{+}$Calcd for $\mathrm{C}_{22} \mathrm{H}_{26} \mathrm{~N}_{2} \mathrm{O}_{5} \mathrm{Na}, 421.1739$; Found: 421.1740. 
Di-t-butyl 2-(cyclohexylimino)-2,5-dihydro-5-oxo-1-phenyl-1H-pyrrole-3,4-dicarboxylate (3c). Orange powder, yield 88\%, $0.40 \mathrm{~g}, \mathrm{mp} 66-68{ }^{\circ} \mathrm{C}$; IR $(\mathrm{KBr})\left(v_{\max }, \mathrm{cm}^{-1}\right): 1755$ and 1723 $\left(2 \mathrm{C}=\mathrm{O}\right.$ of ester), $1660(\mathrm{C}=\mathrm{O}) .{ }^{1} \mathrm{H} \mathrm{NMR}\left(400.1 \mathrm{MHz}, \mathrm{CDCl}_{3}\right): \delta_{\mathrm{H}} 1.60$ and $1.63(18 \mathrm{H}, 2 \mathrm{~s}$, $\left.2 \mathrm{C}\left(\mathrm{CH}_{3}\right)_{3}\right), 1.34-1.78\left(10 \mathrm{H}, \mathrm{m}, 5 \mathrm{CH}_{2}\right), 3.77(1 \mathrm{H}, \mathrm{m}, \mathrm{N}-\mathrm{CH}), 7.11-7.53(5 \mathrm{H}, \mathrm{m}, \mathrm{Ar}-\mathrm{H}) .{ }^{13} \mathrm{C}$ NMR $\left(125.7 \mathrm{MHz}, \mathrm{CDCl}_{3}\right): \delta_{\mathrm{C}} 24.2,25.6$ and $33.3\left(5 \mathrm{CH}_{2}\right.$ of cyclohexyl), 28.1 and $28.6\left(2 \mathrm{C}\left(\mathrm{CH}_{3}\right)_{3}\right)$, $58.5(\mathrm{~N}-\mathrm{CH}), 81.0$ and $83.0\left(2 \mathrm{OCMe}_{3}\right), 124.8,126.3,127.3,128.7,135.5$ and $137.3\left(\mathrm{C}_{\text {arom }}\right.$ and $\left.\mathrm{C}=\mathrm{C}_{\text {pyrrole ring }}\right), 148.1(\mathrm{C}=\mathrm{N}), 148.8(\mathrm{C}=\mathrm{O}), 159.1$ and $159.4(2 \mathrm{C}=\mathrm{O}$ of ester $) . \mathrm{MS}$, we $(\%)=454$ (M+ 4), 397 (10), 324 (40), 280 (45), 251 (13), 198 (28), 119 (19), 83 (45), $77(19), 57$ (100), 55 (80), 41 (48); Anal. Calcd for $\mathrm{C}_{26} \mathrm{H}_{34} \mathrm{~N}_{2} \mathrm{O}_{5}$ (454.56): C, 68.70; H, 7.54; N, 6.16\%. Found: C, $68.75 ; \mathrm{H}, 7.60 ; \mathrm{N}, 6.20 \%$.

Dimethyl 2-(t-butylimino)-2,5-dihydro-5-oxo-1-phenyl-1 $H$-pyrrole-3,4-dicarboxylate (3d). Yellow powder, yield 95\%, $0.33 \mathrm{~g}, \mathrm{mp} 59-61^{\circ} \mathrm{C}$; IR $(\mathrm{KBr})\left(v_{\max }, \mathrm{cm}^{-1}\right): 1752$ and $1729(2 \mathrm{C}=\mathrm{O}$ of ester), $1666(\mathrm{C}=\mathrm{O}) .{ }^{1} \mathrm{H} \mathrm{NMR}\left(400.1 \mathrm{MHz}^{\mathrm{C}} \mathrm{CDCl}_{3}\right)$ : $\delta_{\mathrm{H}} 1.32\left(9 \mathrm{H}, \mathrm{s}, \mathrm{CMe}_{3}\right), 3.92$ and $3.95(6 \mathrm{H}$, $\left.2 \mathrm{~s}, 2 \mathrm{OCH}_{3}\right), 7.28-7.36(5 \mathrm{H}, \mathrm{m}, \mathrm{Ar}-\mathrm{H}) .{ }^{13} \mathrm{C} \mathrm{NMR}\left(100.6 \mathrm{MHz}, \mathrm{CDCl}_{3}\right): \delta_{\mathrm{C}} 28.9\left(\mathrm{CMe}_{3}\right), 52.1$ and 52.2 (2OMe), 55.8 ( $\left.\mathrm{N}-\underline{\mathrm{CMe}}_{3}\right), 123.1,125.2,127.6,134.6,137.3,143.2\left(\mathrm{C}_{\text {arom }}\right.$ and $\left.\mathrm{C}=\mathrm{C}_{\text {pyrrole ring }}\right)$, $147.6(\mathrm{C}=\mathrm{N}), 148.0(\mathrm{C}=\mathrm{O}), 159.4$ and $159.6(2 \mathrm{C}=\mathrm{O}$ of ester $)$. $\mathrm{MS}, w_{e}(\%)=345\left(\mathrm{M}^{+}+1,14\right)$, $344\left(\mathrm{M}^{+}, 6\right), 329$ (100), 313(75), 256 (58), 197 (91), 119 (29), 77 (17), 57 (43); HRMS (ESI): $m z$ $=[\mathrm{M}+\mathrm{Na}]^{+}$Calcd for $\mathrm{C}_{18} \mathrm{H}_{20} \mathrm{~N}_{2} \mathrm{O}_{5} \mathrm{Na}, 367.1270$; Found: 367.1267.

Diethyl 2-(t-butylimino)-2,5-dihydro-5-oxo-1-phenyl-1H-pyrrole-3,4-dicarboxylate (3e). Yellow powder, yield $82 \%, 0.31 \mathrm{~g}, \mathrm{mp} 63-65^{\circ} \mathrm{C}$; IR $(\mathrm{KBr})\left(v_{\max }, \mathrm{cm}^{-1}\right): 1744$ and $1721(2 \mathrm{C}=\mathrm{O}$ of ester), $1668(\mathrm{C}=\mathrm{O}) .{ }^{1} \mathrm{H}$ NMR $\left(400.1 \mathrm{MHz}, \mathrm{CDCl}_{3}\right)$ : $\delta_{\mathrm{H}} 1.28\left(9 \mathrm{H}, \mathrm{s}, \mathrm{CMe}_{3}\right), 1.38$ and $1.40(6 \mathrm{H}, 2 \mathrm{t}$, $\left.{ }^{3} \mathrm{~J}_{\mathrm{HH}}=7.0 \mathrm{~Hz}, 2 \mathrm{CH}_{3}\right), 4.35$ and $4.39\left(4 \mathrm{H}, 2 \mathrm{q},{ }^{3} \mathrm{~J}_{\mathrm{HH}}=7.0 \mathrm{~Hz}, 2 \mathrm{OCH}_{2}\right), 7.19-7.40(5 \mathrm{H}, \mathrm{m}, \mathrm{Ar}-\mathrm{H})$. ${ }^{13} \mathrm{C}$ NMR $\left(100.6 \mathrm{MHz}, \mathrm{CDCl}_{3}\right): \delta_{\mathrm{C}} 13.9$ and $14.0\left(2 \mathrm{CH}_{3}\right), 30.04\left(\mathrm{CMe}_{3}\right), 56.8\left(\mathrm{~N}-\underline{\mathrm{CMe}}_{3}\right), 62.3$ and $62.4\left(2 \mathrm{OCH}_{2}\right), 124.3,126.2,128.6,135.6,138.0,144.5\left(\mathrm{C}_{\text {arom }}\right.$ and $\left.\mathrm{C}=\mathrm{C}_{\text {pyrrole ring }}\right), 147.4$ $(\mathrm{C}=\mathrm{N}), 151.1(\mathrm{C}=\mathrm{O}), 160.3$ and $160.8\left(2 \mathrm{C}=\mathrm{O}\right.$ of ester). $\mathrm{MS}$, we $(\%)=373\left(\mathrm{M}^{+}+1,14\right), 372\left(\mathrm{M}^{+}\right.$, 5), 357 (80), 270 (62), 243 (30), 197 (75), 119 (28), 77 (24), 57 (40); Anal. Calcd for $\mathrm{C}_{20} \mathrm{H}_{24} \mathrm{~N}_{2} \mathrm{O}_{5}$ (372.41): C, 64.50; H, 6.50; N, 7.52\%. Found: C, 64.43; H, 6.58; N, 7.44\%.

Di-t-butyl 2-(t-butylimino)-2,5-dihydro-5-oxo-1-phenyl-1 $H$-pyrrole-3,4-dicarboxylate (3f). Pale yellow powder, yield 85\%, $0.36 \mathrm{~g}, \mathrm{mp} 57-59{ }^{\circ} \mathrm{C}$; IR $(\mathrm{KBr})\left(v_{\max }, \mathrm{cm}^{-1}\right): 1744$ and 1719 $\left(2 \mathrm{C}=\mathrm{O}\right.$ of ester), $1662(\mathrm{C}=\mathrm{O}) .{ }^{1} \mathrm{H}$ NMR $\left(400.1 \mathrm{MHz}, \mathrm{CDCl}_{3}\right): \delta_{\mathrm{H}} 1.32\left(9 \mathrm{H}, \mathrm{s}, \mathrm{N}-\mathrm{C}\left(\mathrm{CH}_{3}\right)_{3}\right), 1.61$ and $1.68\left(18 \mathrm{H}, 2 \mathrm{~s}, 2 \mathrm{OC}\left(\mathrm{CH}_{3}\right)_{3}\right), 7.03-7.39(5 \mathrm{H}, \mathrm{m}, \mathrm{Ar}-\mathrm{H}) .{ }^{13} \mathrm{C} \mathrm{NMR}\left(100.6 \mathrm{MHz}, \mathrm{CDCl}_{3}\right): \delta_{\mathrm{C}}$ $27.7\left(\mathrm{~N}-\mathrm{C}\left(\underline{\mathrm{CH}}_{3}\right)_{3}\right), 28.1$ and $30.1\left(2 \mathrm{OC}\left(\underline{\mathrm{CH}}_{3}\right)_{3}\right), 56.4\left(\mathrm{~N}-\underline{\mathrm{CMe}}_{3}\right), 82.0$ and $84.2\left(2 \mathrm{OCMe}_{3}\right), 124.1$, 125.8, 128.6, 129.1, 134.6, $138.4\left(\mathrm{C}_{\text {arom }}\right.$ and $\left.\mathrm{C}=\mathrm{C}_{\text {pyrrole ring }}\right), 148.7(\mathrm{C}=\mathrm{N}), 149.8(\mathrm{C}=\mathrm{O}), 159.1$ and $159.5\left(2 \mathrm{C}=\mathrm{O}\right.$ of ester). MS, $m$ e $(\%)=429\left(\mathrm{M}^{+}+1,27\right), 428\left(\mathrm{M}^{+}, 5\right), 357(7), 301(77), 283(5)$, 242 (79), 215 (13), 119 (9), 77 (10), 57 (100); Anal. Calcd for $\mathrm{C}_{24} \mathrm{H}_{32} \mathrm{~N}_{2} \mathrm{O}_{5}$ (428.52): C, 67.27; H, 7.53 ; N, 6.54\%. Found: C, 67.35; H, 7.61; N, 6.65\%.

Dimethyl 2-(2,6-dimethylphenylimino)-2,5-dihydro-5-oxo-1-phenyl-1H-pyrrole-3,4dicarboxylate $\mathbf{( 3 g})$. Yellow powder, yield $84 \%, 0.33 \mathrm{~g}, \mathrm{mp} 107-109{ }^{\circ} \mathrm{C}$; IR $(\mathrm{KBr})\left(v_{\max }, \mathrm{cm}^{-1}\right)$ : 1745 and $1688\left(2 \mathrm{C}=\mathrm{O}\right.$ of ester), $1594(\mathrm{C}=\mathrm{O}) .{ }^{1} \mathrm{H}$ NMR $\left(400.1 \mathrm{MHz}, \mathrm{CDCl}_{3}\right): \delta_{\mathrm{H}} 2.13(6 \mathrm{H}, \mathrm{s}$, $\left.2 \mathrm{CH}_{3}\right), 3.99$ and $4.02\left(6 \mathrm{H}, 2 \mathrm{~s}, 2 \mathrm{OCH}_{3}\right), 6.95-7.36(8 \mathrm{H}, \mathrm{m}, 2 \mathrm{Ar}-\mathrm{H}) .{ }^{13} \mathrm{C} \mathrm{NMR}(100.7 \mathrm{MHz}$, 
$\left.\mathrm{CDCl}_{3}\right): \delta_{\mathrm{C}} 17.8\left(2 \mathrm{CH}_{3}\right), 53.3$ and $53.4\left(2 \mathrm{OCH}_{3}\right), 118.1,121.7,122.2,123.8,127.6,128.8,128.9$, $130.0\left(\mathrm{C}_{\text {arom }}\right), 136.9$ and $139.8\left(\mathrm{C}=\mathrm{C}_{\text {pyrrole ring }}\right), 148.8(\mathrm{C}=\mathrm{N}), 152.6(\mathrm{C}=\mathrm{O}), 158.8$ and 159.9 $(2 \mathrm{C}=\mathrm{O}$ of ester $)$. MS, $m / e(\%)=393\left(\mathrm{M}^{+}+1,16\right), 392\left(\mathrm{M}^{+}, 62\right), 377(5), 361(8), 301(51), 274$ (6), 212 (64), 167 (6), 119 (17), 93 (100), 91 (14), 77 (61), 59 (8); HRMS (ESI): $m z=[\mathrm{M}+\mathrm{Na}]^{+}$ Calcd for $\mathrm{C}_{22} \mathrm{H}_{20} \mathrm{~N}_{2} \mathrm{O}_{5} \mathrm{Na}, 415.1270$; Found: 415.1271 .

Di-t-butyl 2-(2,6-dimethylphenylimino)-2,5-dihydro-5-oxo-1-phenyl-1 $H$-pyrrole-3,4dicarboxylate (3h). Red powder, yield $81 \%, 0.39 \mathrm{~g}, \mathrm{mp} 92-94{ }^{\circ} \mathrm{C}$; IR $(\mathrm{KBr})\left(v_{\max }, \mathrm{cm}^{-1}\right): 1735$ and $1696\left(2 \mathrm{C}=\mathrm{O}\right.$ of ester), $1594(\mathrm{C}=\mathrm{O}) .{ }^{1} \mathrm{H}$ NMR $\left(400.1 \mathrm{MHz}, \mathrm{CDCl}_{3}\right): \delta_{\mathrm{H}} 1.47$ and $1.51(18 \mathrm{H}$, $\left.2 \mathrm{~s}, 2 \mathrm{C}\left(\mathrm{CH}_{3}\right)_{3}\right), 2.25\left(6 \mathrm{H}, \mathrm{s}, 2 \mathrm{CH}_{3}\right), 7.01-7.61(8 \mathrm{H}, \mathrm{m}, 2 \mathrm{Ar}-\mathrm{H}) .{ }^{13} \mathrm{C} \mathrm{NMR}\left(100.6 \mathrm{MHz}, \mathrm{CDCl}_{3}\right): \delta_{\mathrm{C}}$ $18.3\left(2 \mathrm{CH}_{3}\right), 27.3$ and $27.5\left(2 \mathrm{C}\left(\underline{C H}_{3}\right)_{3}\right), 82.5$ and $82.7\left(2 \mathrm{OCMe}_{3}\right), 118.1,119.4,121.7,123.6$, 126.8, 127.7, 128.8, $130.8\left(\mathrm{C}_{\text {arom }}\right), 135.5$ and $139.8\left(\mathrm{C}=\mathrm{C}_{\text {pyrrole ring }}\right), 150.0(\mathrm{C}=\mathrm{N}), 152.6(\mathrm{C}=\mathrm{O})$, 160.2 and $161.3\left(2 \mathrm{C}=\mathrm{O}\right.$ of ester). $\mathrm{MS}, m e(\%)=477\left(\mathrm{M}^{+}+1,7\right), 476\left(\mathrm{M}^{+}, 20\right), 420(6), 375(6)$, 347 (13), 275 (14), 245 (13), 212 (37), 119 (12), 105 (10), 93 (100), 91 (12), 77 (33), 57 (49); HRMS (ESI): $m z=[\mathrm{M}+\mathrm{Na}]^{+}$Calcd for $\mathrm{C}_{28} \mathrm{H}_{32} \mathrm{~N}_{2} \mathrm{O}_{5} \mathrm{Na}, 499.2209$; Found: 499.2208.

\section{Acknowledgments}

We gratefully acknowledge financial support from the Research Council of University of Sistan and Baluchestan.

\section{References}

1. Swinbourne, J. F.; Hunt, H. J.; Klinkert, G. Adv. Heterocycl. Chem. 1987, 23, 103.

2. (a) Domling, A.; Ugi, I. Angew. Chem., Int. Ed. 2000, 39, 3168. (b) Ugi, I.; Werner, B.; Domling, A. Molecules 2003, 8, 53.

3. Ullmanns Encycl. Tech. Chem. 1957, 9, 1.

4. Ozaki, S. Chem. Rev. 1972, 72, 457.

5. L'abbé, G. Synthesis 1987, 525.

6. Ulrich, H. Cycloaddition Reactions of Heterocumulenes; Academic: New York: London, 1967; pp 122.

7. Ulrich, H. Acc. Chem. Res. 1969, 2, 186.

8. Winterfeldt, E.; Schumann, D.; Dillinger, H. J. Chem. Ber. 1969, 102, 1656.

9. Oakes, T. R.; David, H. G.; Nagel, F. J. Am. Chem. Soc. 1969, 91, 4761.

10. Takisawa, T.; Obata, N.; Suzuki, Y.; Yanagida, T. Tetrahedron Lett. 1969, 10, 3407.

11. Ugi, I. Isonitrile Chemistry; Academic: London, 1971.

12. Ugi, I. Angew. Chem., Int. Ed. Engl. 1982, 21, 810.

13. Yavari, I.; Maghsoodlou, M. T. J. Chem. Res. 1998, 386. 
14. Nair, V.; Rajesh, C.; Vinod, A. U.; Bindu, S.; Sreekanth, A. R.; Mathen J. S.; Balagopal, L. Acc. Chem. Res. 2003, 36, 899.

15. Shaabani, A.; Hossein-Rezayan, A.; Ghasemi, S.; Sarvary, A. Tetrahedron Lett. 2009, 50, 1456.

16. Teimouri, M. B.; Mansouri, F.; Bazhrang, R. Tetrahedron 2010, 66, 259.

17. Alizadeh, A.; Hosseinpour, R. Synthesis 2009, 16, 2733.

18. Shaabani, A.; Sarvary, A.; Hossein-Rezayan, A.; Keshipour, S. Tetrahedron 2009, 65, 1.

19. Alizadeh, A.; Rostamnia, S.; Zohreh, N.; Bijanzadeh, H. Monatsh Chem. 2008, 139, 49.

20. Maghsoodlou, M. T.; Hazeri, N.; Habibi-Khorassani, S. M.; Marandi, G.; Nassiri, M. Synth. Commun. 2005, 35, 2771.

21. Maghsoodlou, M. T.; Hazeri, N.; Habibi-Khorassani, S. M.; Marandi, G.; Nassiri, M. J. Heterocyclic Chem. 2006, 43, 481.

22. Maghsoodlou, M. T.; Habibi-Khorasani, S. M.; Hazeri, N.; Heydari, R.; Marandi, G.; Nassiri, M. J. Chem. Res. 2006, 220.

23. Maghsoodlou, M. T.; Hazeri, N.; Habibi-Khorassani, S. M.; Solimani, V.; Marandi, G.; Razmjoo, Z. J. Chem. Res. 2008, 198.

24. Hazeri, N.; Maghsoodlou, M. T.; Habibi-Korassani, S. M.; Marandi, G.; Khandan-Barani, K.; Ziyadini, M.; Aminkhani, A. Arkivoc 2007, (i), 173.

25. Hazeri, N.; Maghsoodlou, M. T.; Habibi-Korassani, S. M.; Ziyadini, M.; Marandi, G.; KhandanBarani, K.; Bijanzadeh, H. R. Arkivoc 2007, (xiii), 34.

26. Maghsoodlou, M. T.; Marandi, G.; Hazeri, N.; Habibi-Korassani, S. M.; Mirzaei, A. A. Mol. Divers 2010, 14,1 .

27. Marandi, G.; Maghsoodlou, M. T.; Hazeri, N.; Heydari. R.; Habibi-Korassani, S. M.; Ebrahimi, A.; Poor, S. M.; Mahdiabad, H. H.; Nassiri, M.; Kabiri, R. Heteroatom. Chem. 2010, 21, 228. 